\title{
A HIGH GRADIENT SUPERCONDUCTING QUADRUPOLE FOR A LOW CHARGE STATE ION LINAC
}

\author{
J.W. Kim, K.W. Shepard, and J.A. Nolen, Argonne National Laboratory, Argonne, IL 60439 USA
}

A superconducting quadrupole magnet has been designed for use as the focusing element in a low charge state linac proposed at Argonne. The expected field gradient is $350 \mathrm{~T} / \mathrm{m}$ at an operating current of $53 \mathrm{~A}$, and the bore diameter is $3 \mathrm{~cm}$. The use of rare earth material holmium for pole tips provides about $10 \%$ more gradient than iron pole tips. The design and the status of construction of a prototype singlet magnet is described.

\section{INTRODUCTION}

A radioactive ion beam facility proposed at ANL [1] [2] requires accelerating ions with $\mathrm{q} / \mathrm{m} \geq 1 / 66$ and $\beta(=v / \mathrm{c})$ $\geq 0.004$ through a superconducting drift tube linac. In the present ATLAS linac, the transverse focusing elements are 8 tesla superconducting solenoids, which are used for beams with a $\mathrm{q} / \mathrm{m} \geq 1 / 10$ and $\beta \geq 0.008$ [3]. However, the low charge state beams contempleted here require much stronger focusing; magnetic solenoid elements would need to produce axial fields higher than 15 tesla for adequate transverse focusing. More effective focusing can be obtained with either magnetic or electric quadrupoles. We ruled out electrostatic quadrupoles because of uncertainty both in holding the required voltage and in operation at $4 \mathrm{~K}$. Considering magnetic elements, permanent magnet quadrupoles have been used for drift-tube linacs, but in the present application would need complete shielding to avoid trapped flux and excessive rf loss in the neighboring superconducting cavities. Also, permanent magnet quadrupoles would not provide an adequate gradient for the required aperature.

A series of beam optics calculations has shown that quadrupole triplets with a gradient of $350 \mathrm{~T} / \mathrm{m}$, a bore radius of $3 \mathrm{~cm}$, and an overall length of approximately $20 \mathrm{~cm}$ can provide adequate transverse focusing. The design is simplified by the fact that harmonic components in quadrupole field are not so detrimental to beam quality in the present application as in recirculating machines. Beam dynamics calculations indicate that non-quadrupole harmonics with an integrated effect up to $1 \%$ at a radius of 1 $\mathrm{cm}$ are tolerable. The design of a prototype magnet which can meet these requirements is discussed below.

\section{DESIGN OF A PROTOTYPE QUADRUPOLE}

Design options for superconducting quadrupoles include $\cos 2 \theta$ or superferric types. Construction methods for forming $\cos 2 \theta$ coils with NbTi wires have been established, but such high field quality (and high construction cost) is not needed in this linac application. Also, it is desireable to use a small size of wire to reduce the refrigeration budget, which would make a $\cos 2 \theta$ winding even more difficult. For these reasons, we have chosen a magnet design based on simple racetrack coils with pole tips.

A schematic view of the prototype is shown in Fig.1. The configuration of the prototype is similar to a superconducting quadrupole developed at LANL [4], but with a modified winding to accomodate a substantially increased bore radius. Each of the four coils is composed of three blocks to increase the coil size and to keep the coil configuration simple. In fact, coil currents could be more effectively used if the coil were expanded closer to the field region, maintaining a $\cos 2 \theta$ current distribution (e.g. ref.[5]), but then the coil winding becomes more difficult and expensive.

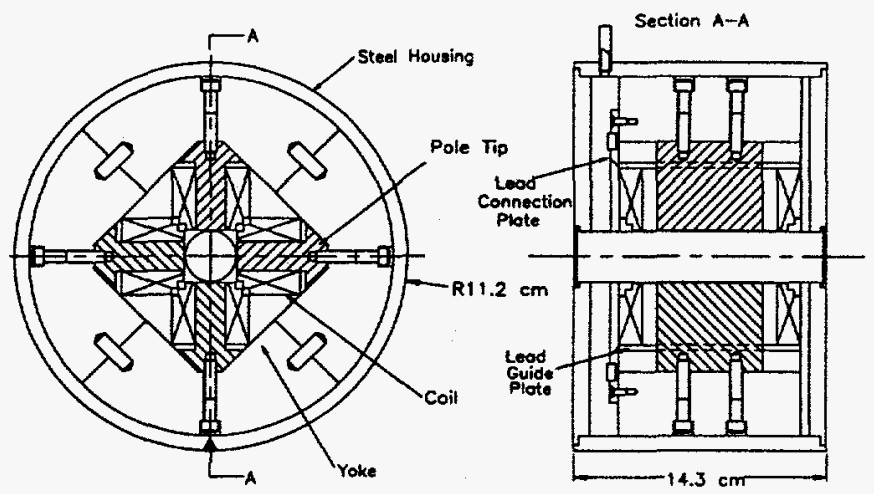

Figure 1: A schematic view of a prototype quadrupole

The pole tips are made of rare earth material holmium to enchance the magnetic flux concentration. This technique has been utilized for high field solenoids [6] as well as for the LANL prototype quad; the saturation magnetization of holmium at $4.2 \mathrm{~K}$ is 3.8 tesla. The pole tip has a simple flat face since it is saturated at the operating field. Flat pole tips have been employed for room temperature quadrupoles in drift tube linacs [7]. The prototype is a quadrupole singlet with a length equal to the longest element of the proposed triplet, since the longer quadrupole magnet could be more subject to wire motion problems. The inner corner of the section of the coil near the pole face has a small notch $(1.3 \times 2 \mathrm{~mm})$ removed to reduce the peak field on the conductor.

The magnetic fields were numerically calculated first using the OPERA-2d code [8] to optimize the pole tip width and minimize the higher order harmonics. This was a compromise process, trading-off useful aperature against low harmonic content. At $350 \mathrm{~T} / \mathrm{m}$, the 12 th pole is about 0.1 


\section{DISCLAIMER}

Portions of this document may be illegible in electronic image products. Images are produced from the best available original document. 
Table 1: Design parameters of a prototype quadrupole

\begin{tabular}{|c|c|}
\hline Gradient & $350 \mathrm{~T} / \mathrm{m}$ \\
Operating current & $53 \mathrm{~A}$ at $350 \mathrm{~T} / \mathrm{m}$ \\
Current density in coil & $47,000 \mathrm{~A} / \mathrm{cm}^{2}$ \\
Pole tips & holmium $(99.6 \%)$ \\
Inductance & $0.55 \mathrm{H}$ \\
No of turns & 3800 \\
Yoke & $\mathrm{SAE} 1008$ steel \\
\hline Wire & $\mathrm{NbTi}$ \\
Diameter & $0.305 \mathrm{~mm}$ \\
Cu/Sc & 1.35 \\
Critical current & $57 \mathrm{~A}$ at $6.3 \mathrm{tesla}, 4.2 \mathrm{~K}$ \\
Insulation & Formvar, 0.0127 mm thick \\
\hline
\end{tabular}

$\%$ of quadrupole field, and the 20th pole is less than $0.1 \%$. In Fig.2, the field lines are plotted for a quadrant of the quadrupole. The B-H curve of holmium used in the numerical calculation was taken from ref.[6]. At $350 \mathrm{~T} / \mathrm{m}$ the gradient gain from using holmium is about $35 \mathrm{~T} / \mathrm{m}$ higher than for iron pole tips. Major design parameters of the prototype are listed in Table.1.

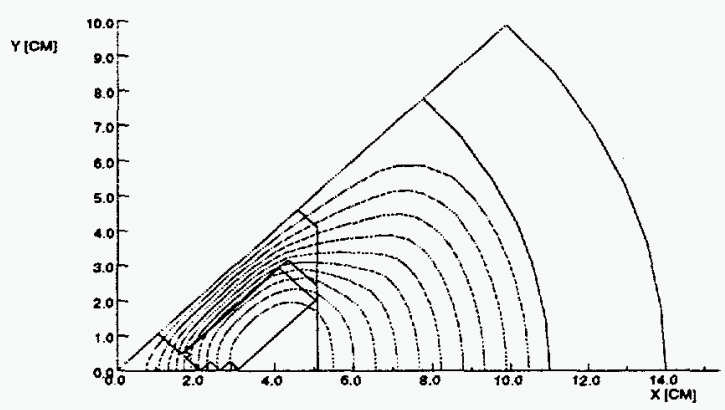

Figure.2: Magnetic field lines for a quadrant of the quadrupole, as calculated with the OPERA-2d code

Since the prototype quadrupole length is short, 3-d effects are important to field quality. A 3-d finite element program TOSCA [8] was used to calculate fringe fields. The field profile of a transvese field component $\left(\mathrm{B}_{t}\right)$ is plotted in Fig. 3 at a radius of $1 \mathrm{~cm}$ and $350 \mathrm{~T} / \mathrm{m}$. The effective magnetic length is $7.6 \mathrm{~cm}$.

The effect of harmonic field components on the beam is determined by the integrated gradient along the quadrupole. The integrated gradient error defined in the equation below is used to specify the field quality;

$$
\Delta I_{G}(r, z)=\frac{\int_{0}^{z} B_{t}\left(r, \theta_{\max }\right) d z-\int_{0}^{z} B_{t}\left(r, \theta_{\min }\right) d z}{\int_{0}^{z} B_{t}\left(r, \theta_{\min }\right) d z}
$$

where $\theta_{\max }$ is an angle which gives a maximum of the integral, and $\theta_{\min }$ for a minimum at a radius of $r$. The dashed line in Fig. 3 shows $\Delta I_{G}(\mathrm{r}, \mathrm{z})$ as a function of $\mathrm{z}$ at $r=1 \mathrm{~cm}$. Note that the intgerated error is reduced as the beam traverses the coil ends: this effect has been used to design high quality $\cos 2 \theta$ quadrupoles. The intergated harmonic content increases for lower fields; it rises to $0.6 \%$ at 200 $\mathrm{T} / \mathrm{m}$, and further to $2.5 \%$ at $100 \mathrm{~T} / \mathrm{m}$ when the current density is $6400 \mathrm{~A} / \mathrm{cm}^{2}$.

The coil end design has two important issues: 1) harmonic errors, 2) enchancement of field on the conductor. To reduce both effects, the coil ends could be spread out using spacers [9]. However, such control of the end effect was not necessary for the present application. The harmonic error is reduced as the beam passes through the quadrupole as shown in Fig.3, although some field enchancement is unavoidable. The highest field on conductor is about $6.3 \mathrm{~T}$ at the ends compared to $6.0 \mathrm{~T}$ in the straight section, as calculated with TOSCA.

The coil is an orderly winding, with insulation sheets embedded between layers and impregnated with epoxy. The winding is placed directly on the holmium pole, eliminating several steps needed when a winding form is used. The winding load is about $8 \mathrm{~N}$, producing a winding tension of $9 \times 10^{3} \mathrm{~N} / \mathrm{cm}^{2}$. The coil is continously wound without splice, and G-10 spacers are used to fill the rectangular gaps during winding.

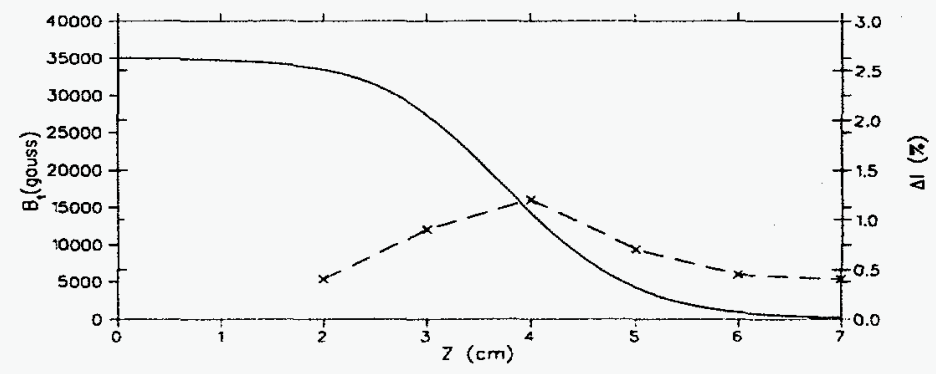

Figure 3: The field profile of $\mathrm{B}_{t}$ at $\mathrm{r}=1 \mathrm{~cm}$ (solid line) and the integrated gradient error (dashed line) are shown as a function of axial distance. The axial distance is from the center of the quadrupole.

\section{COIL FORCE AND QUENCH CALCULATIONS}

The electromagnetic forces on the straight section of the coil are depicted in Fig.3 for an excitation of $350 \mathrm{~T} / \mathrm{m}$. Although the winding tension prevents wire motion on the curved coil ends and although the coil is rather short, the straight section still needs a support. Without support the coil may expand as much as $0.3 \mathrm{~mm}$ at $350 \mathrm{~T} / \mathrm{m}$ in the middle of the straight section, as calculated using the deflection formula for the case of supported edges in ref. [10]. The straight section is simply supported by stainless steel spacers. To avoid motion of lead wires from the coils, the leads are fixed onto the lead connection plates made of G-10, as noted in Fig.1.

An estimate of quench voltage at the junction between quenched and normal coils is about 400 volts as calculated with the program QUENCH [11]. Although such a voltage is not difficult to insulate, when 2 or 3 singlets of a given triplet are wired in series quench voltages of $1 \mathrm{kV}$ or more 
can appear. For triplet elements, a compromise between quench voltage and the number of connecting leads into cryostat will need to be made.

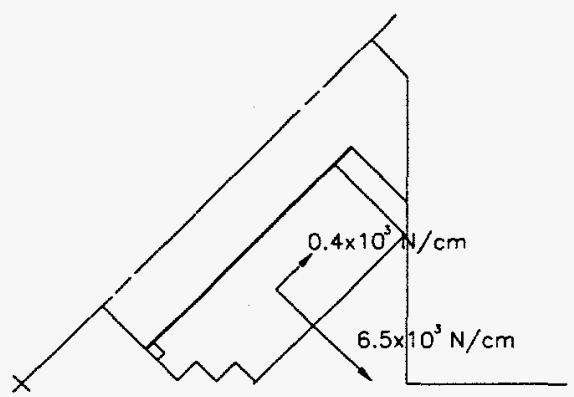

Figure 4: Forces on the straight section of the coil at $350 \mathrm{~T} / \mathrm{m}$

\section{STATUS AND CONCLUSIONS}

A prototype superconducting quadrupole with a $3 \mathrm{~cm}$ aperture has been designed for use in a low charge state injector linac for ATLAS. The design field gradient of 350 $\mathrm{T} / \mathrm{m}$ can be achieved when the operating current is $91 \%$ of the critical current at $4.2 \mathrm{~K}$. This tight design margin demands a high quality winding. 3 -d field calculations showed that the integrated field gradient error is $0.45 \%$ at 350 $\mathrm{T} / \mathrm{m}$ and less than $1 \%$ in a wide range of coil excitations.

Construction of a prototype singlet is well advanced. The winding is being carried out by Cryomagnetics Inc. The winding and testing of the first coil is expected to be completed in May 1995. Following testing of the first coil, the remaining 3 coils for the prototype singlet will be wound. Final assembly and field measurements will be carried out at Argonne. The prototype is designed to allow testing different coil supporting structures with little modification. The final design will be optimized for economic production of several dozen units.

\section{Acknowledgment}

We would like to thank Drs. L. Turner and K. Thompson of the Advanced Photon Source at Argonne National Laboratory for their generous help in using TOSCA, and Dr. A. Zeller at Michigan State University for providing the B-H data.

\section{References}

[1] J.A. Nolen, Accelerator Complex for a Radioactive Ion Beam Facility at ATLAS, in the proceedings of this conference

[2] K.W. Shepard and J.W. Kim, A Low-Charge-State Injector Linac for ATLAS, in the proceedings of this conference

[3] A.H. Jaffey et al., Nucl Instr Meth, 121 (1974) 413

[4] D.B.Barlow et al., Nucl Instr Meth, A313 (1992) 311

[5] G. Ambrosio, et al., Proceedings of 1994 European Particle Accelerator Conference, (1994) 2268

[6] W.Schauer and F.Arendt, Cryogenics, 23 (1983) 562
[7] I.M.Kapchinskiy, Theory of Resonance Linear Accelerators, 81 (1985) harwood academic publishers

[8] OPERA-2d and TOSCA are products of Vector Fields, Oxford, England

[9] S.Caspi, M.Helm, and L.J.Laslett, Magnetic Field in the End Region of the SSC Quadrupole Magnet, LBL29819, (1991)

[10] R.D.Cook and W.C.Young, Advanced Mechanics of Materials, Macmillan Publishing Co. (1985)

[11] M.N.Wilson, program QUENCH, Rutherford Laboratory Report, RHEL/M151 (1968)

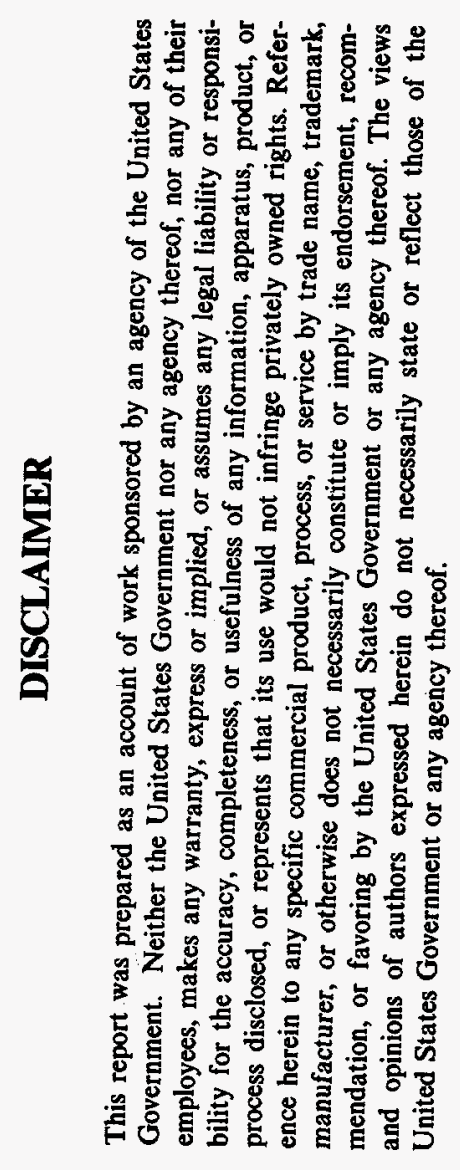

